\title{
Erratum
}

\section{Efficient Syntheses of $\beta$-Amino- $N$-acylbenzotriazoles and Cinnamides through Regioselective 1,4- or 1,2-Addition of Amines to $\mathrm{N}$-Cinnamoylbenzotriazoles}

Xiaoxia Wang,* Xuefei Zou, Jian Li, Qinghong Hu Synlett 2005, 3042-3046.

In the original article the addition mode of the reaction given in Scheme 1 had been misinterpreted as normal 1,4-addition. Therefore the structure of compound $\mathbf{3}$ shown in Scheme 1 was incorrect. Further investigation, which was stimulated by Gandelman and Jacobsen, ${ }^{1}$ revealed that a different product $\mathbf{3}$ is received. Below the correct Scheme 1 is shown.

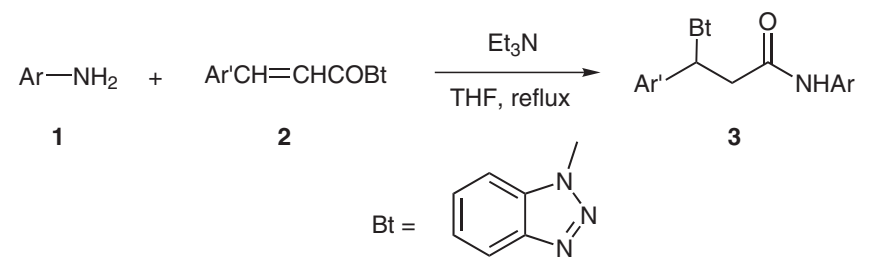

Scheme 1

The structure of product 3a was ascertained by recent single crystal X-ray diffraction analysis.

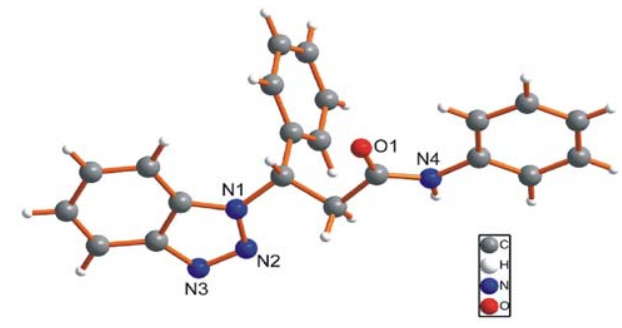

Further investigation is in progress to have a deeper insight into this reaction.

(1) Gandelman, M.; Jacobsen, E. N. Angew. Chem. Int. Ed. 2005, 44, 2393.

The authors apologize for the errors. 